\title{
Neuroprotective Effects and Related Mechanisms of Echinacoside in MPTP-Induced PD Mice
}

\author{
Zhen-Nian Zhang ${ }^{l}, *$ \\ Zhen Hui ${ }^{1} *$ \\ Chang Chen' \\ Yan Liang' \\ Li-Li Tang' \\ Su-Lei Wang' \\ Cheng-Cheng $\mathrm{Xu}{ }^{\prime}$ \\ Hui Yang' \\ Jing-Si Zhang ${ }^{2}$ \\ Yang Zhao'
}

'Department of Neurology, Nanjing Hospital of Chinese Medicine Affiliated to Nanjing University of Chinese Medicine, Nanjing, 210022, People's Republic of China; ${ }^{2}$ Department of Neurology, Shuguang Hospital Affiliated to Shanghai University of Traditional Chinese Medicine, Shanghai, 201203, People's Republic of China

*These authors contributed equally to this work

\begin{abstract}
Objective: To explore the neuroprotective effect and the related mechanisms of echinacoside (ECH) in 1-methyl-4-phenyl-1,2,3,6-tetrahydropyridine (MPTP)-induced Parkinson's disease (PD) mice.

Methods: Parkinson's disease is induced in mice by MPTP and the neurobehaviors of mice in different groups are observed. Then, immunohistochemistry and Western blot analysis are adopted to measure the expression of tyrosine hydroxylase (TH) and $\alpha$-synuclein in the substantia nigra (SN). The content of dopamine (DA) and other neurotransmitters in the brain is detected by high-performance liquid chromatography. The expression of nerve growth factors and inflammatory factors in SN in mice in each group is measured by quantitative polymerase chain reaction. Finally, the expression of oxidative stress-related parameters in each group is measured.
\end{abstract}

Results: Compared with the model group, the pole-climbing time among mice in the moderate and high-dose $\mathrm{ECH}$ groups is significantly reduced $(\mathrm{P}<0.01)$. The rotarod staying time, as well as fore and hind-limb strides, shows a significant increase $(\mathrm{P}<0.01)$, as does spontaneous activity $(\mathrm{P}<0.01)$. Moreover, the expression levels of TH, DA, glial cell linederived neurotrophic factor, and brain-derived neurotrophic factor in $\mathrm{SN}$ in mice show significant increases in these two groups $(\mathrm{P}<0.01)$. The content of superoxide dismutase, catalase, and glutathione peroxidase indicates significant increases in the low, moderate, and high-dose ECH groups $(\mathrm{P}<0.01)$, and the content of MDA was reduced $(\mathrm{P}<0.01)$. In the high-dose ECH group, the expression of interleukin (IL) 6 and tumor necrosis factor- $\alpha$ is significantly reduced $(\mathrm{P}<0.01)$, while the expression of IL-10 shows a marked increase $(\mathrm{P}<$ $0.01)$ alongside a decrease in the expression of $\alpha$-synuclein $(\mathrm{P}<0.01)$.

Conclusion: Echinacoside improves neurobehavioral symptoms in PD mice and significantly increases the expression of $\mathrm{TH}$ and DA. The neuroprotective effect potentially correlates with anti-inflammation and anti-oxidation actions, promotes the expression of nerve growth factor, and reduces the accumulation of $\alpha$-synuclein.

Keywords: Parkinson's disease, MPTP, echinacoside, $\alpha$-synuclein, oxidative stress, neuroprotection, BDNF, GDNF, IL-6, TNF- $\alpha$, IL-10

\section{Introduction}

Parkinson's disease (PD) is a chronic and progressive neurodegenerative disease. A typical pathological feature of PD is the formation of Lewy bodies, in which $\alpha$ synuclein and ubiquitin are the primary components. ${ }^{1,2}$

Although the pathogenesis of PD has not been fully clarified, ever more evidence indicates that environmental factors, oxidative stress, mitochondrial dysfunction, down-regulation of neurotrophic factors, immune inflammation, excitatory amino-acid toxicity, calcium overload, and other pathology mechanisms
Department of Neurology, Nanjing Hospital of Chinese Medicine Affiliated to Nanjing University of Chinese Medicine,

No. 157 of Daming Road, Qinhuai

District, Nanjing, 210022, People's

Republic of China

$\mathrm{Tel} / \mathrm{Fax}+86$ I385I4984I8

Email zhao_dr66@163.com 
interact and participate in the occurrence or development of PD. Studies suggested that risk factors, such as increased oxidative stress and mitochondrial dysfunction, can lead to misfolding and abnormal aggregation of $\alpha$ synuclein. This in turn may aggravate oxidative stress and mitochondrial dysfunction. These factors may interact with one another and continuously amplify the effect of injury, eventually leading to progressive degeneration and death of dopaminergic neurons. ${ }^{3}$

Considering that no drug can prevent the progression of PD at present, combined with the pathogenesis and core pathological characteristics of PD, targeting $\alpha$-synuclein may indicate an important direction for finding drugs that can delay the disease's progression and probe the neuroprotective effects. ${ }^{4}$

Recent studies showed that echinacoside $(\mathrm{ECH})$ has a wide range of pharmacological effects, including antiinflammatory, antioxidative, and neuroprotective functions; additionally, it may contribute to improving learning and memory, provide hepatic protection, and immune regulation, and provide anti-tumor effects. ${ }^{5}$ Existing studies conducted by the present study group also confirmed that ECH may be able to improve abnormal gait in the 1-methyl-4-phenyl-1,2,3,6-tetrahydropyridine (MPTP)induced PD mice model. It may also significantly reduce the expression of $\mathrm{Bax} / \mathrm{Bcl}-2$ and inhibit the reduction of dopaminergic neurons in the striatum, as well as inhibit the activation of microglia and new types of glial cells. ${ }^{6-10} \mathrm{In}$ the pathogenesis of $\mathrm{PD}$, the activation of microglia can secrete many neurotoxic substances such as chemokines, inflammatory factors, and reactive oxygen-free radicals, and aggravate damage to the neurons through oxidative stress, inflammation, and the induction of apoptosis. Researchers have found that in the MPTP-induced PD mice model, inhibiting the activation of microglia can prevent the death of dopaminergic neurons. ${ }^{8}$ Cell models showed that ECH may be able to reduce the expression of inflammatory factors induced by 6-hydroxydopamine. ${ }^{11}$

However, there are few reports on the effects of ECH and its related therapeutic mechanisms for the clearance of $\alpha$-synuclein, the core pathological product in PD. In the present study, the elimination of $\alpha$-synuclein was further studied by combining existing studies, considering the neuroprotective mechanisms of ECH (anti-inflammatory, anti-oxidative stress), and the enhancement of glial cellderived nerve growth factor (GDNF) and brain-derived nerve growth factor (BDNF).

\section{Materials and Apparatus}

\section{Experimental Animals}

The experimental group comprised 108 eight-week-old male C57BL/6J mice (specific-pathogen-free (SPF) grade) weighing 20-22 g. Four mice were kept per cage at $22^{\circ} \mathrm{C}-25^{\circ} \mathrm{C}$. The mice were provided by the Shanghai Center for Laboratory Animals, Chinese Academy of Sciences, housed at the Nanjing University of Chinese Medicine (Nanjing, China). Animals were treated humanely according to the National Institutes of Health guidelines in an SPF-grade room with a temperature of $22^{\circ} \mathrm{C}-$ $25^{\circ} \mathrm{C}, 55 \%$ relative humidity, and under a 12 -h circadian rhythm. The mice had free access to food and water.

\section{Primary Reagents}

The primary reagents used in the current study were as follows: MPTP (Sigma-Aldrich, Product number: M0896), mouse anti-tyrosine hydroxylase (TH) monoclonal antibody (Sigma-Aldrich Company), Alexa Fluor 555 antirabbit antibody (Biyuntian Institute of Biotechnology), Alexa Fluor 488 anti-rabbit antibody (Biyuntian Institute of Biotechnology), selegiline (Sigma-Aldrich), antihorseradish peroxidase (HRP)-goat anti-rabbit secondary antibody (Biyuntian Institute of Biotechnology), rabbit polyclonal $\alpha$-synuclein antibody (Cell Signaling Technology), HRP-goat anti-mice secondary antibody (Biyuntian Institute of Biotechnology), and ECH (Chengdu Linghangzhe Biotechnology Co., Ltd.).

Preparation of MPTP: $100 \mathrm{mg}$ MPTP powder was added to $10 \mathrm{~mL} 0.9 \%$ normal saline, thoroughly shaken and mixed to avoid light, and then divided into $101.5 \mathrm{~mL}$ Eppendorf tubes and stored at $-20^{\circ} \mathrm{C}$. Prior to use, the solution was diluted once to achieve a final concentration of $5 \mathrm{mg} / \mathrm{mL}$.

\section{Experimental Methods Grouping of the Experimental Animals and Medication Grouping of Experimental Animals}

The $108 \mathrm{C} 57 \mathrm{BL} / 6 \mathrm{~J}$ mice were randomly divided into six groups with 18 mice in each. The details of grouping were as follows: the normal control group (the normal saline group, MPTP group, low-dose ECH group [the EL group], moderatedose $\mathrm{ECH}$ group [the EM group], high-dose ECH group [the EH group], and the selegiline group [the SL group]). 


\section{Modeling of the Experimental Animals and Medication}

The MPTP dose for intraperitoneal injection was $30 \mathrm{mg} /$ $\mathrm{kg} / \mathrm{d}$ for seven consecutive days. The selegiline gavage dose was $1 \mathrm{mg} / \mathrm{kg} / \mathrm{d}$. The dose of ECH in EL, EM, and EH groups was $10 \mathrm{mg} / \mathrm{kg} / \mathrm{d}, 20 \mathrm{mg} / \mathrm{kg} / \mathrm{d}$, and $30 \mathrm{mg} / \mathrm{kg} / \mathrm{d}$, respectively. Among these groups, the mice in the normal saline group were intragastrically administered with $0.1 \mathrm{~mL}$ of saline and injected intra-peritoneally with $0.1 \mathrm{~mL}$ of saline during the modeling period. The gavage was started in the three-dose ECH groups and the SL group, 3 days before the MPTP injection, and the mice continued to receiving gavage for 7 days after the termination of the MPTP injection.

\section{Neurobehavioral Observations The Pole Test}

Created based on the literature ${ }^{11}$ (with minor modifications), the self-made device for the pole test is described as follows: The device was a PV tube that was $55 \mathrm{~cm}$ long, $1 \mathrm{~cm}$ in diameter with a 2-cm-diameter spherical projection at the top of the device as an attachment point for mice. The device was covered with black tape to prevent the mice from slipping. A mouse was placed on the spherical protruding point with its head upward during the test. The time from placing the mouse at the top of the bar to its head turning downwards (T-turn), and the total time from placing the mouse at the top of the bar to it climbing to the bottom of the tube to land (T-total) were recorded.

\section{Spontaneous Activity}

Spontaneous activity, also known as "open field activity", is a common indicator for detecting rare activity following MPTP injury. ${ }^{12}$ In the present study, an open field experiment video analysis system was adopted. The specific operations were as follows: Prior to conducting the experiment, four mice of the same group were put into an observation box (length $25 \mathrm{~cm}$, width $25 \mathrm{~cm}$, and height $25 \mathrm{~cm}$ ) to adapt for $30 \mathrm{~min}$. They were then continuously video recorded for $30 \mathrm{~min}$. In the 30-min observation time window, the number of vertical movements (rearing number) was counted separately at three discontinuous intervals of $5 \mathrm{~min}$. The video analysis system automatically analyzed the trajectory of the mice's activities and obtained a 30-min total distance (total distance) as an indicator of horizontal movement.

\section{Gait Analysis}

Based on the literature, ${ }^{13}$ a self-made gait analysis device was used. This comprised a wooden walkway (length $42 \mathrm{~cm}$, width $4.5 \mathrm{~cm}$, height $12 \mathrm{~cm}$ ), one end of which was open while the opposite end led to a squirrel cage. The squirrel cage was covered with a black cloth. Blue ink was dipped onto the bottom of a mouse's feet. The mouse was then placed at the open end of the walkway. The mouse naturally moved to the end with the squirrel cage covered with black cloth. The three longest fore and hind-limb strides were measured, respectively, and the average value was derived.

\section{The Rotarod Test}

A mouse was placed on a variable-speed rotarod, the speed of which was adjusted to increase from 4 rounds/min to 40 rounds/min within an overall time of $5 \mathrm{~min}$. The time from the beginning of the rotarod rotation to the mouse dropping from the rotarod each time was recorded as the latency to fall. ${ }^{14}$

\section{Determination of the Content of Neurotransmitters in the Striatum by High-Performance Liquid Chromatography Electrochemistry Detection}

Couloehemlll 5300 electrochemical chromatography was adopted. The detection means were 5020 and 504 LB cells, with a chromatographic column of MD-150 (3.0 $\times$ $150 \mathrm{~mm} ; 3$ um), a mobile MDTM-2 phase, and a sensitivity level of $2 \mathrm{uA}$. The flow rate was set to $0.6 \mathrm{~mL} / \mathrm{min}$, the potential of the electrode was set to 220 $\mathrm{mV}$, and the column temperature was set to $30^{\circ} \mathrm{C}$. The injection volume was $20 \mathrm{ul}$ (first diluted 10 times). The content of the following neurotransmitters was detected: dopamine (DA), dihydroxyphenylacetic acid (DOPAC), high vanillic acid, norepinephrine, 5-hydroxytryptamine, and 5-hydroxyindoleacetic acid.

\section{The Immunohistochemical Staining of Tyrosine Hydroxylase and $\alpha$-Synuclein}

Following the behavioral tests, mice ( $\mathrm{n}=6$ per group) were euthanized under deep anesthesia ( $10 \%$ chloral hydrate) and perfused with $0.9 \%$ sodium chloride followed by $4 \%$ paraformaldehyde through the left cardiac ventricle. Brains were quickly removed and post-fixed for $24 \mathrm{~h}$, then transferred to 
phosphate-buffered saline (PBS) containing 30\% sucrose at $4^{\circ} \mathrm{C}$ until they sank. Tissue was embedded in paraffin, and coronal sections $(30 \mu \mathrm{m})$ were cut through the substantia nigra pars compacta ( $\mathrm{SNpc} ;-2.8$ to $-3.8 \mathrm{~mm}$, caudal to the bregma) using a sledge microtome. The sections were dewaxed and hydrated, and the endogenous peroxidase was quenched with $0.3 \%$ hydrogen peroxide $\left(\mathrm{H}_{2} \mathrm{O}_{2}\right)$ for $30 \mathrm{~min}$. After heat-induced antigen retrieval, the slides were incubated with $0.5 \%$ Triton-X10, then with $5 \%$ bovine serum albumin for $30 \mathrm{~min}$ each. The sections were then incubated with rabbit anti- $\alpha$-synuclein antibody or rabbit anti-TH antibody overnight at $4{ }^{\circ} \mathrm{C}$. After three 5-min washes in PBS, the sections were incubated in biotinylated goat anti-rabbit antibody for $1 \mathrm{~h}$, followed by avidin-biotin complex for $30 \mathrm{~min}$. Peroxidase activity was visualized with 3,3-diaminobenzidine. For quantitative analysis of TH and $\alpha$-synucleinimmunoreactive neurons, 10 consecutive coronal sections across the center of the SNpc were counted bilaterally using Image-Pro Plus (v.6.0) in a blinded fashion.

The brain slices were observed by stereographic microscopy; the immunohistochemical positive cells of TH and $\alpha$-synuclein in SN of the midbrain were calculated using the stereographic counting system. According to the rat brain atlas, the target region of $\mathrm{SN}$ of the midbrain was delineated; the counting frame was $100 \times 100 \mu \mathrm{m}$. Cell counts of both indexes were performed under a 20 $\mathrm{x}$ objective lens. Cell counts were analyzed using STEREO Investigator (v.7.0) (Micro Bright Field. Inc., Williston, VT, USA) software to calculate the total number of positive cells.

\section{The Detection of Protein by Western Blot Analysis}

The SNpc protein was extracted ( $\mathrm{n}=6$ mice per group), separated using $12-15 \%$ sodium dodecyl sulfatepolyacrylamide gel electrophoresis, and transferred to polyvinylidene fluoride membranes. The membranes were blocked with $5 \%$ nonfat milk for $1.5 \mathrm{~h}$ at room temperature and incubated with antibodies against $\mathrm{TH}, \alpha$ synuclein, and GAPDH at $4{ }^{\circ} \mathrm{C}$ overnight. The next day, membranes were rinsed with tris-buffered saline containing $0.1 \%$ Tween ${ }^{\circledR} 20$ and hybridized with HRP-conjugated anti-rabbit immunoglobulin G for $1.5 \mathrm{~h}$. Proteins were visualized using enhanced chemiluminescence, and immunoreactive bands were analyzed with image analysis software (Gel Doc 1000-UV; Bio-Rad, Richmond, CA) to calculate the optical density of $\alpha$-synuclein and $\mathrm{TH}$ bands normalized to GADPH.

\section{The Detection of Oxidative Stress-Related Parameters}

Approximately $0.1 \sim 0.2 \mathrm{~g}$ SN tissue was rinsed and weighed in ice-cold normal saline and put into a $5 \mathrm{~mL}$ homogenizing tube. With water bathing, the corresponding volume of the homogenization medium $(0.86 \%$ normal saline) was added into the glass homogenizing tube according to the ratio of weight $(\mathrm{g})$ : volume $(\mathrm{mL})=1: 9$. The tissue was fully ground to render a $10 \%$ homogenate. The prepared homogenate was centrifuged at $2500 \mathrm{rpm}$ for $15 \mathrm{~min}$, and the supernatant was taken for detection. The protein concentration was determined following the operating steps of the total protein detection kit of the Nanjing Jiancheng Institute of Bioengineering. The detection wavelength was $570 \mathrm{~nm}$. With pre-treatment and detection of the protein concentration, the content of superoxide dismutase (SOD), malondialdehyde (MDA), catalase (CAT), and glutathione peroxidase (GSH-Px) was detected in the samples.

\section{The Polymerase Chain Reaction Procedures}

Total RNA was extracted from the frozen tissue samples of SNpc with TRIzol (Sigma, USA) and converted to cDNA using the PrimeScript RT reagent kit (Takara, Japan). According to the manufacturer's instructions, expression levels of the target gene, mRNA, were detected by realtime quantitative polymerase chain reaction (q-PCR; Applied Biosystems 7500, USA) using a SYBR ${ }^{\circledR}$ Premix Ex Taq II kit (Takara, Japan). The thermocycling conditions started at $95^{\circ} \mathrm{C}$ for $30 \mathrm{~s}$, followed by 40 denaturation q-PCR cycles at $95^{\circ} \mathrm{C}$ for $5 \mathrm{~s}$ and an extension at $60^{\circ} \mathrm{C}$ for 34 s. Melting curves were performed at the end of each cycle to ascertain the purity of the q-PCR products. The primer sequences for GAPDH and the target genes were as follows:

GDNF (5'GGGACUCUAAGAUGAAGUUdTdT3';3'dT dTCCCUGAGAUUCUACUUCAA5'), BDNF ( $5^{\prime}$ GGUCAC AGUCCUAGAGAAAdTdT3'; 3'dTdTCCAGUGUCAGGAUCUCUUU5'), tumor necrosis factor- $\alpha$ (TNF- $\alpha$ ): (5'GC СТCTTCTCATTCCTGCTTG3';5'CTGATGAGAGGGAGGCCATT3'), IL-6 (5'TGGGAAATCGTGGAAATGAG 
3'; 5'CTCTGAAGGACTCTGGCTTTG3'), IL-10 (5'CAA CATACTGCTAACCGACTCCT3';5'TGAGGGTCTTCAGCTTCTCAC $\left.3^{\prime}\right)$, GAPDH (5'-GGT TGT CTC CTG CGA CTT CA-3'; $3^{\prime}$-CCT CAT TCT TTG GGA CCT GGT-5').

\section{Statistical Analysis}

All measurement data in the present study were expressed in the form of means \pm standard error, and statistical analysis was processed using GraphPad Prism 5.0 software (GraphPad Software Inc., San Diego, CA); P $<0.05$ was considered statistically significant. One-way analysis of variance was used to test the statistical differences, and comparisons between multiple groups were analyzed by Tukey's multiple group comparisons.

\section{The Experimental Results}

\section{The Observation of the Neurobehavioral Symptoms in MPTP-Induced PD Mice}

\section{The Pole Test}

The T-turn and the T-Total in the MPTP group were significantly longer compared with those in the Normal saline group $(\mathrm{P}<0.01)$. The T-turn and T-Total in the EH group significantly decreased compared with those in the MPTP group $(\mathrm{P}<0.01)$. Compared with the SL group, there was no significant difference in the above two indicators $(\mathrm{P}>0.05)$ (as shown in Figure 1A).

\section{The Spontaneous Activity}

The rearing number within five minutes, and the total distance within 30 minutes, all significantly decreased in the MPTP group compared with those in the Normal saline group $(\mathrm{P}<0.01)$. The above two indicators in the EM group, EH group, and SL group significantly increased compared with those in the MPTP group $(\mathrm{P}<0.01)$. There was no significant difference in the above two indicators between the EH group and the SL group (P > 0.05) (as demonstrated in Figure 1B).

\section{The Gait Analysis}

The fore and hind limb strides significantly reduced in the MPTP group compared with those in the Normal saline group $(\mathrm{P}<0.01)$. The fore and hind limb strides in the EM group, EH group, and SL group significantly increased compared with those in the MPTP group $(\mathrm{P}<0.01)$. There was no significant difference in the above two indicators between the EH group and the SL group (P > 0.05) (as shown in Figure 1C).

\section{The Rotarod Test}

The staying time on the rotarod, also known as the latency to fall, significantly reduced in the MPTP group compared with that in the Normal saline group $(\mathrm{P}<0.01)$. The latency to fall in the EM group, EH group, and SL group significantly increased compared with that in the MPTP group $(\mathrm{P}<0.01)$. There existed no significant difference in the latency to fall between the EH group and the SL group $(P>0.05)$ (as illustrated in Figure 1D).

\section{Detection of Neurotransmitters in the Striatum in MPTP-Induced PD Mice by HPLC-ECD}

Compared with the Normal saline group, the content of DA in the MPTP group significantly decreased $(\mathrm{P}<0.01)$. The contents of DA in the three-dose groups of ECH and the SL group were significantly higher than that in the MPTP group $(\mathrm{P}<0.01$ and $\mathrm{P}<0.05$, respectively). The content of DA in the EH group significantly increased compared with those in EL and EM group $(\mathrm{P}<0.01)$. The difference in the content of DA between the EH group and the SL group was of no statistical significance ( $\mathrm{P}>$ 0.05) (as shown in Figure 2A). Compared with the Normal saline group, the content of DOPAC in the MPTP group significantly decreased $(\mathrm{P}<0.01)$. The content of DOPAC in the EM group, EH group, and the SL group were significantly higher than that in the MPTP group ( $\mathrm{P}<$ 0.01 and $\mathrm{P}<0.05$, respectively). The difference in the content of DOPAC between the EH group and the SL group was of no statistical significance $(P>0.05)$ (as demonstrated in Figure 2B). Compared with the Normal saline group, the content of HVA in the MPTP group significantly decreased $(\mathrm{P}<0.01)$. The content of HVA in the EM group, EH group, and the SL group significantly increased compared with that in the MPTP group $(\mathrm{P}<0.01$ and $\mathrm{P}<0.05$, respectively). The difference in the content of HVA between the EH group and the SL group was of no statistical significance $(\mathrm{P}>0.05)$ (as shown in Figure $2 \mathrm{C}$ ). Compared with the Normal saline group, the content of 5 -HT in the MPTP group significantly decreased ( $<<$ 0.01). The contents of 5-HT in the EM group, EH group, and the SL group significantly increased compared with that in the MPTP group $(\mathrm{P}<0.01$ and $\mathrm{P}<0.05$, respectively). The difference in the content of 5-HT among the EL group, EM group, and the EH group was of no statistical significance $(\mathrm{P}>0.05)$ (as illustrated in Figure 2D). Compared with the Normal saline group, the content of 
A
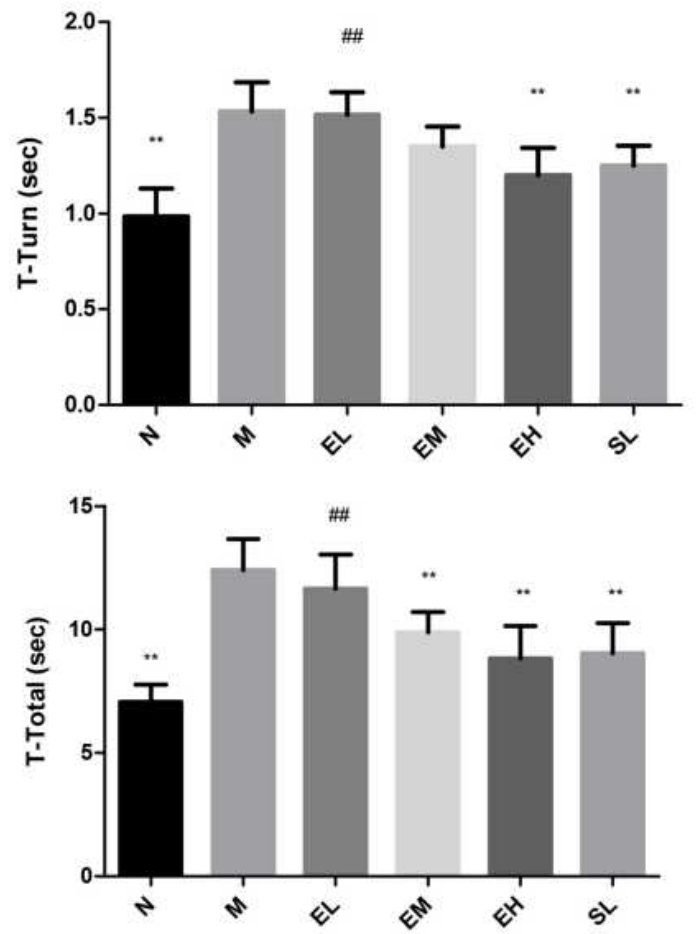

C
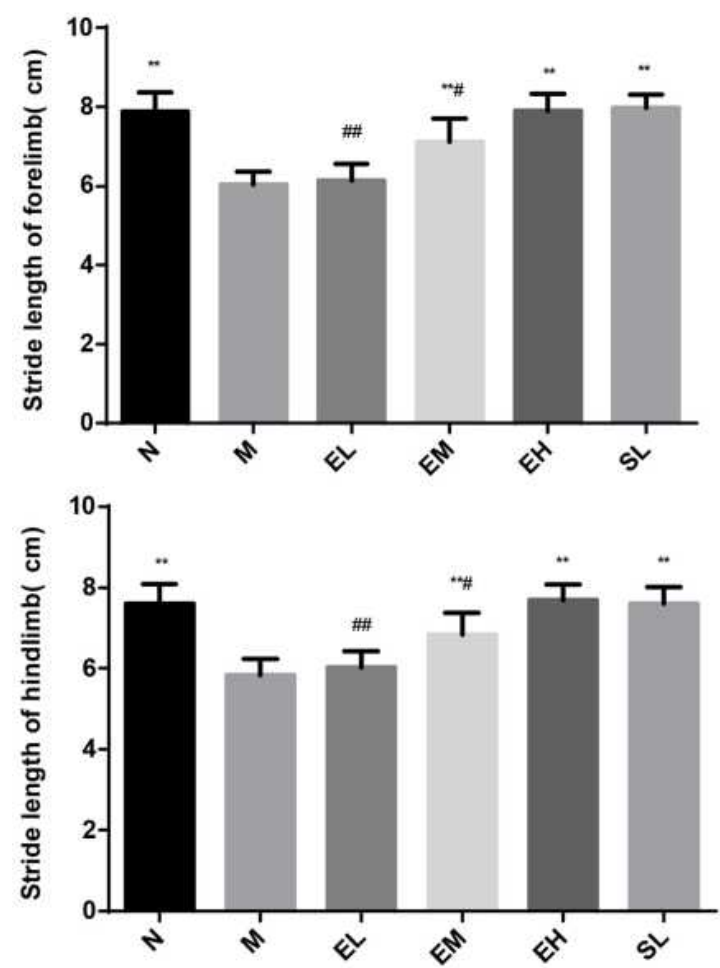

B
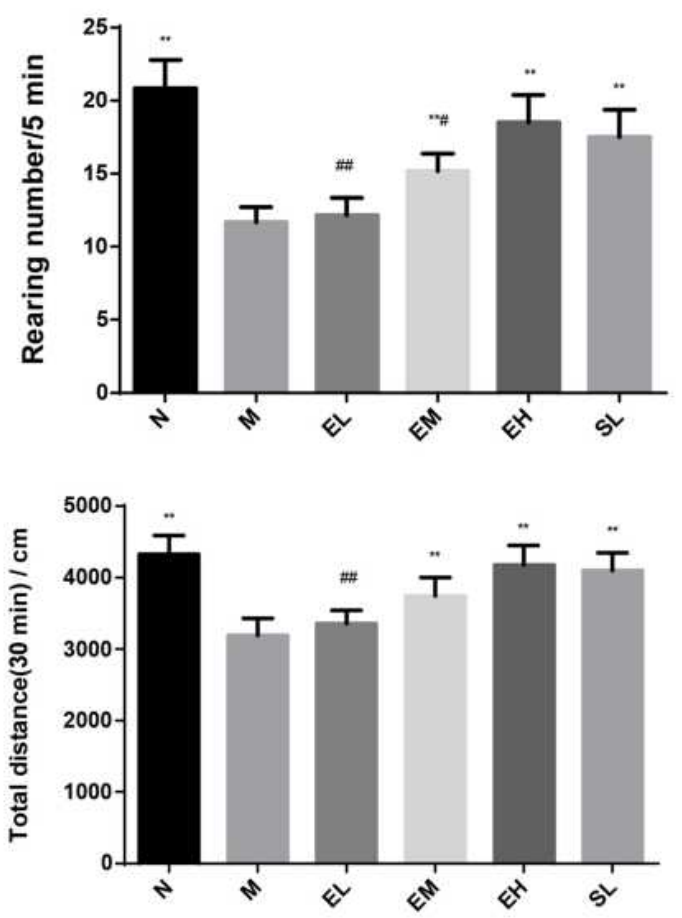

D

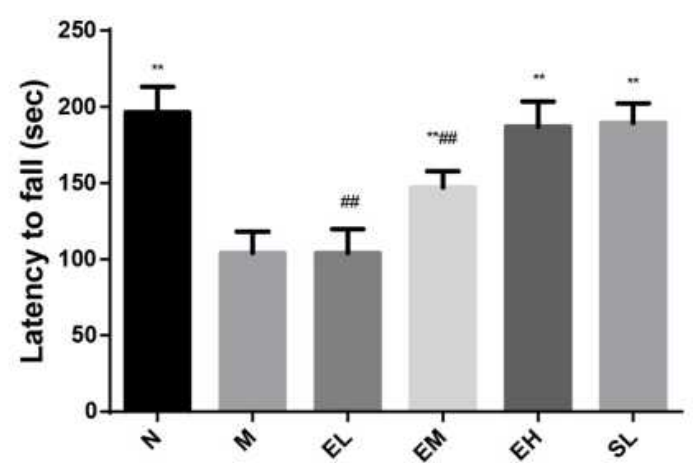

Figure I The neurobehavior in mice of each group. (A) The pole test in mice of each group: Turning time (T-Turn); Total climbing time (T-Total). (B) The spontaneous activity in mice of each group: The erection times within five minutes (Rearing number); The horizontal movement distance within 30 minutes (Total Distance). (C) The gait analysis in mice of each group: The stride length of the fore limb; The stride length of the hind limb. (D) The rotarod in mice of each group: The staying time on the rotarod (also known as the latency to fall). ${ }^{* *} \mathrm{P}<0.01$, compared with the model group; ${ }^{\#} \mathrm{P}<0.05,{ }^{\#} \mathrm{P}<0.01$, compared with the high-dose echinacoside group, $\mathrm{n}=6$. 
A

DA

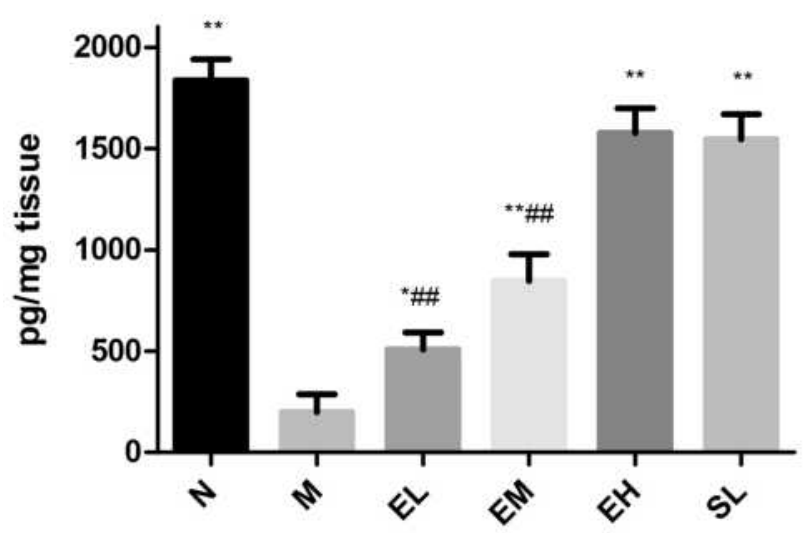

C

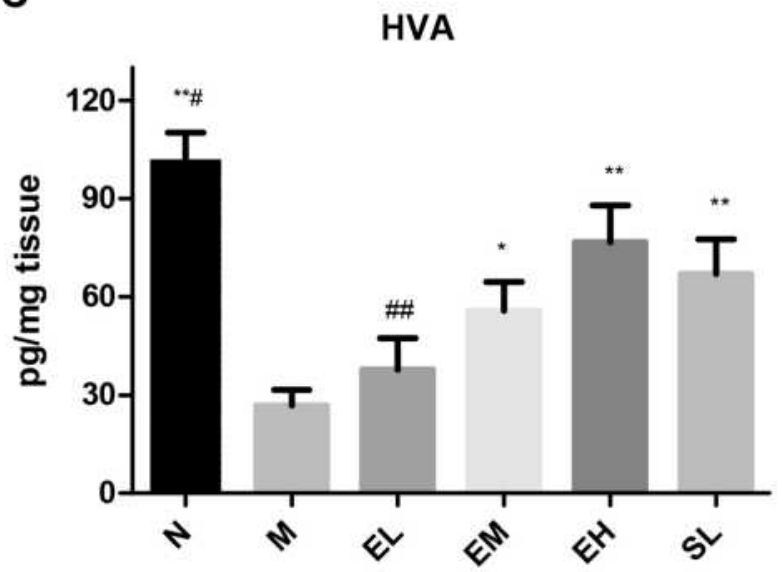

E

5HIAA

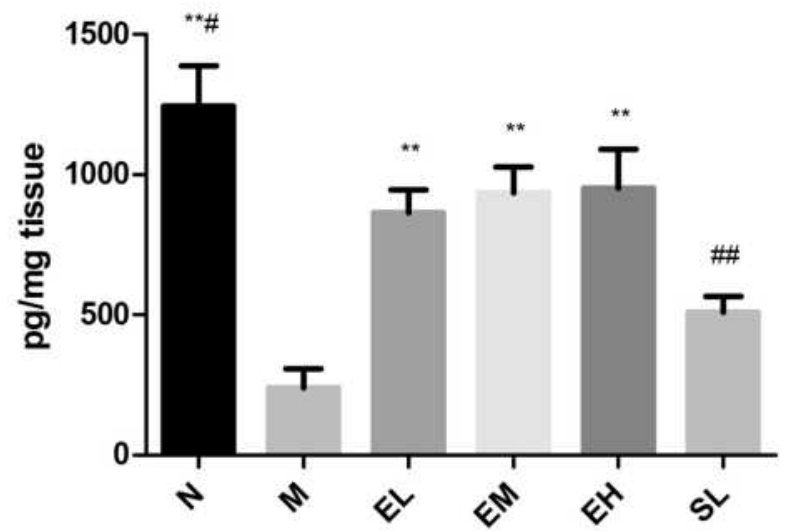

B

DOPAC

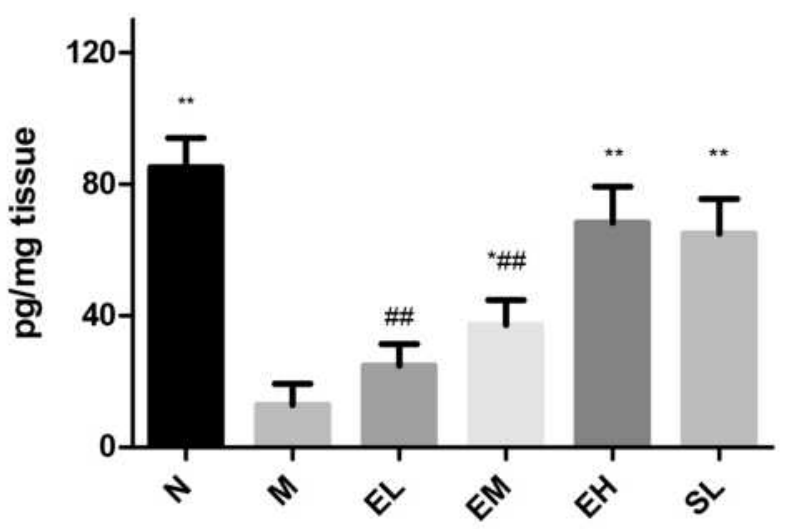

D

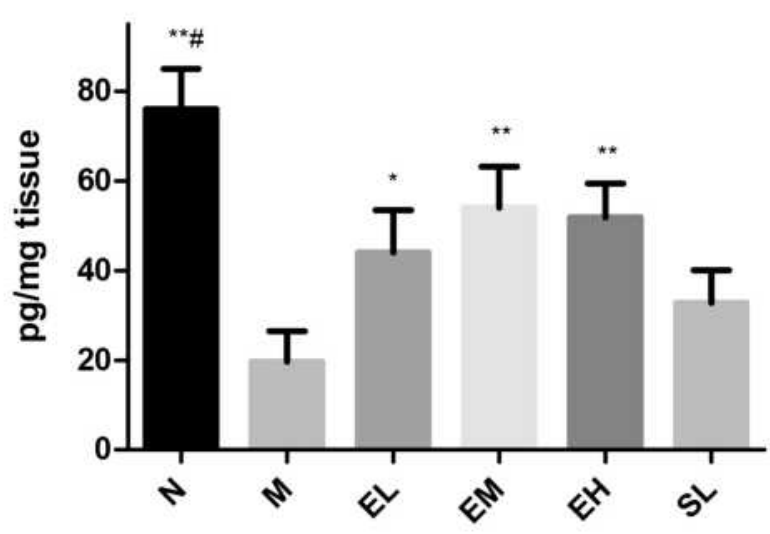

$\mathbf{F}$

NE

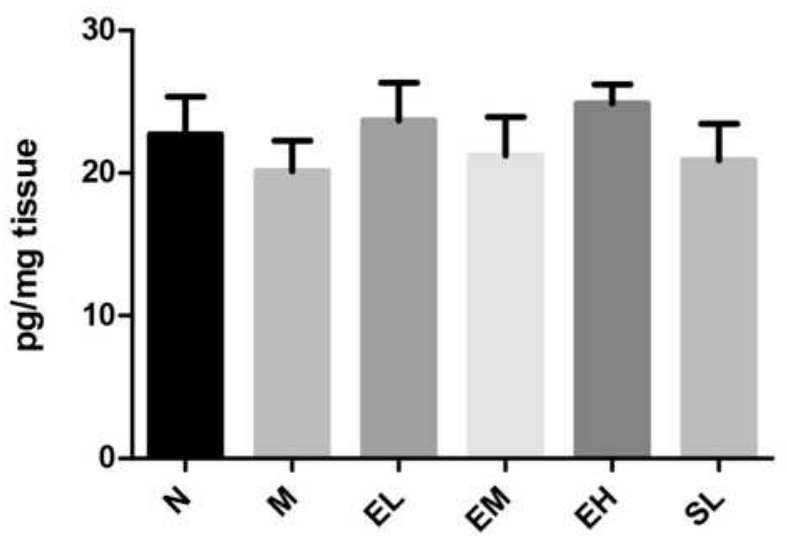

Figure 2 Detection of the neurotransmitter contents in the striatum by the High-performance liquid phase electrochemical method (HPLC-ECD). (A) dopamine (DA); (B) dihydroxyphenylacetic acid (DOPAC); (C) homovanillic acid (HVA); (D) 5-hydroxytryptamine (5-HT); (E) 5-hydroxyindoleacetic acid (5-HIAA); (F) norepinephrine (NE). *P < 0.05, ** $\mathrm{P}<0.0$ I, compared with the model group; ${ }^{\#} \mathrm{P}<0.05,{ }^{\prime \prime} \mathrm{P}<0.0 \mathrm{I}$, compared with the high-dose echinacoside group.

5-HIAA in the MPTP group significantly decreased $(\mathrm{P}<$ 0.01 ). The content of 5-HIAA in the three-dose groups of $\mathrm{ECH}$ was significantly higher than that in the MPTP group $(\mathrm{P}<0.01)$. The content of 5-HIAA in the SL group significantly decreased compared with that in the $\mathrm{EH}$ group $(\mathrm{P}<0.01)$ (as shown in Figure $2 \mathrm{E}$ ). There was no significant statistical difference in NE among different groups $(\mathrm{P}>0.05)$ (as demonstrated in Figure $2 \mathrm{~F})$. 


\section{The Immunohistochemical Staining of TH} and $\alpha$-Synuclein in the Substantia Nigra in MPTP -Induced PD Mice

$\mathrm{TH}$ in the MPTP group was significantly lower than that in the Normal saline group $(\mathrm{P}<0.01)$. TH increased significantly in the EM group, EH group, and the SL group compared with the MPTP group $(\mathrm{P}<0.01$ and $\mathrm{P}<0.05$, respectively). TH was significantly higher in the $\mathrm{EH}$ group than that in EL and EM group $(\mathrm{P}<0.01$ and $\mathrm{P}<0.05$, respectively). Compared with the MPTP group, the number of TH-positive cells in the EM group and EH group increased by $31.66 \%$ and $47.94 \%$, respectively. There was no significant difference in $\mathrm{TH}$ between the EH group and the SL group $(\mathrm{P}>0.05)$. In the MPTP group, the number of $\alpha$-synuclein positive cells in the substantia nigra significantly increased compared with the Normal saline group $(\mathrm{P}<0.01)$. There existed no significant difference in reducing the number of $\alpha$-synuclein positive cells among the EL group, EM group, and the MPTP group (P $>0.05)$. However, compared with the MPTP group, the number of $\alpha$-synuclein positive cells reduced significantly in the EH group and SL group, and the difference was statistically significant $(\mathrm{P}<0.01$ ) (as shown in Figure 3 ).

\section{The Effect of ECH on the Protein} Expression of TH and $\alpha$-Synuclein in the Substantia Nigra in MPTP-Induced PD Mice

The protein expression of TH in the MPTP group was significantly lower than that in the normal saline group $(\mathrm{P}<0.01)$. The protein expressions of $\mathrm{TH}$ in the EM group, EH group, and the SL group were significantly higher than that in the MPTP group $(\mathrm{P}<0.01)$. The protein expression of TH in the EH group was significantly higher than that in EL and EM group ( $\mathrm{P}<0.01)$. Compared with the $\mathrm{SL}$ group, the difference in the protein expression of $\mathrm{TH}$ was not statistically significant $(\mathrm{P}>0.05)$. The protein expression of $\alpha$-synuclein in the MPTP group was significantly higher than that in the Normal saline group ( $\mathrm{P}<0.01)$. The protein expressions of $\alpha$-synuclein in the EL group and EM group were slightly lower than that in the MPTP group, but the difference was of no statistical significance $(\mathrm{P}>0.05)$. Compared with the MPTP group, the protein expressions of $\alpha$-synuclein significantly reduced in the $\mathrm{EH}$ group and SL group, and the differences were statistically significant $(\mathrm{P}<0.01)$ (as illustrated in Figure 4).

\section{$\mathrm{ECH}$ Reduced the Oxidative Stress Injury} in the Substantia Nigra in MPTP-Induced PD Mice

The contents of CAT, GSH-Px, and SOD in the MPTP group were significantly lower than those in the Normal saline group ( $\mathrm{P}<0.05)$. The content of CAT in EL, EM, and EH groups and SL groups was significantly higher than that in the MPTP group $(\mathrm{P}<0.01)$. The contents of CAT, GSH-Px, and SOD in the EH group were all higher than those in EM and EL group $(\mathrm{P}<0.01)$. The differences in the contents were not statistically significant when compared with those in the SL group $(\mathrm{P}>0.05)$ (as shown in Figure 5A-C). The content of MDA in the MPTP group was significantly higher than that in the Normal saline group $(\mathrm{P}<0.01)$. The content of MDA in EL, EM, EH, and SL groups decreased significantly compared with that in the MPTP group $(\mathrm{P}<0.01)$. The reduction of the content of MDA was more significant in the EH group than that in EL and EM group $(\mathrm{P}<0.01)$. There was no significant statistical significance in the $\mathrm{EH}$ group in the reduction of the MDA content when compared with the SL group ( $\mathrm{P}>0.05)$ (as shown in Figure 5D).

\section{ECH Increased the mRNA Expression of GDNF and BDNF in the Substantia Nigra in MPTP-Induced PD Mice}

The mRNA expressions of GDNF and BDNF in the MPTP group were significantly lower than those in the Normal saline group $(\mathrm{P}<0.01)$. The mRNA expressions of GDNF and $\mathrm{BDNF}$ in $\mathrm{EM}, \mathrm{EH}$, and $\mathrm{SL}$ groups significantly increased compared with those in the MPTP group ( $\mathrm{P}<$ 0.01). The mRNA expression of BDNF in the EL group was also significantly higher than that in the MPTP group ( $P$ $<0.01)$. The mRNA expressions of GDNF and BDNF in the EH group increased significantly compared with those in EL and EM group ( $\mathrm{P}<0.01)$ (as shown in Figure 6).

\section{ECH Reduced the mRNA Expression of the Inflammatory Factors in the Substantia Nigra in MPTP-Induced PD Mice}

The mRNA expression of IL-6 in the MPTP group significantly increased compared with that in the Normal saline group $(\mathrm{P}<0.01)$. The mRNA expressions of IL-6 in EM, EH, and SL groups significantly decreased compared with that in the MPTP group $(\mathrm{P}<0.01)$. The reduction of the mRNA expression of IL-6 in the EH 


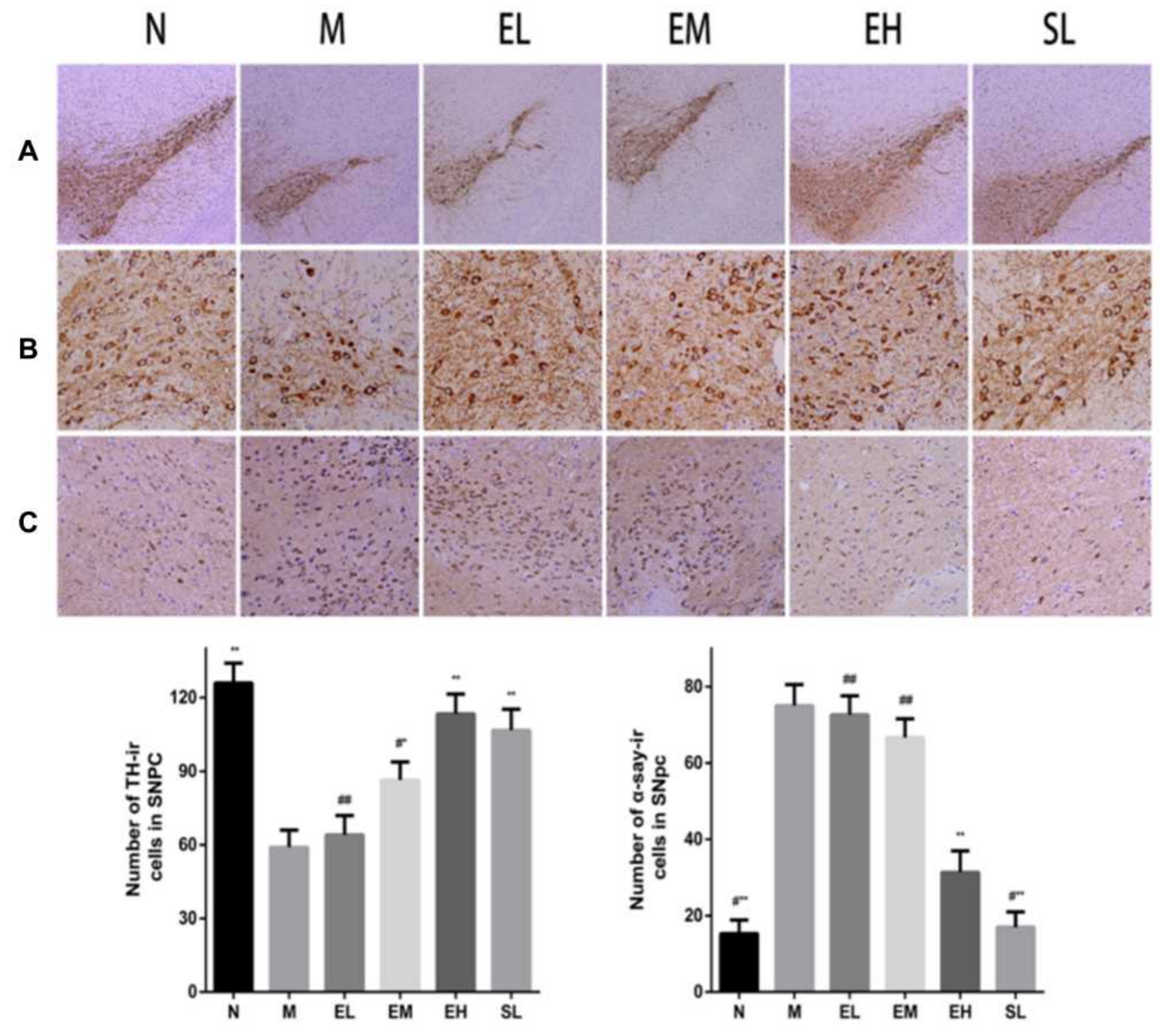

Figure 3 The immunohistochemical staining and quantitative analysis of $\mathrm{TH}$ and $\alpha$-synuclein in the substantia nigra in mice of each group. The upper figure was the immunohistochemical staining of tyrosine hydroxylase $(\mathrm{TH})$ in the substantia nigra in mice $(\mathbf{A}$ and $\mathbf{B})$ and $\alpha$-synuclein $(\mathbf{C})$, the lower figure was the quantitative analysis. $* \mathrm{P}<$ 0.05 , $* * P<0.01$, compared with the model group; ${ }^{\#} \mathrm{P}<0.05,{ }^{\# \#} \mathrm{P}<0.01$, compared with the high-dose echinacoside group.

group was more significant than that in EL and EM group ( $\mathrm{P}<0.01$ and $\mathrm{P}<0.05$, respectively). There was no significant statistical difference compared with the SL group $(\mathrm{P}>0.05)$ (as illustrated in Figure $7 \mathrm{~A})$. The mRNA expression of TNF- $\alpha$ in the MPTP group significantly increased compared with that in the Normal saline group $(\mathrm{P}<0.01)$. Compared with the MPTP group, the mRNA expressions of TNF- $\alpha$ in the EH group and SL group significantly reduced $(\mathrm{P}<0.01)$. There was no statistical difference between the two groups $(\mathrm{P}>0.05)$ (as shown in Figure 7B). The mRNA expression of IL-10 in the MPTP group significantly decreased compared with that in the Normal saline group $(\mathrm{P}<0.01)$.
Compared with the MPTP group, the mRNA expressions of IL-10 in EM, EH, and SL groups significantly increased $(\mathrm{P}<0.01)$. There was no statistical difference among the three groups $(\mathrm{P}>0.05)$ (as demonstrated in Figure 7C).

\section{Discussion}

The MPTP model is widely used by researchers because it can selectively damage the dopaminergic neuron pathway in $\mathrm{SN}$ and striatum and can simulate most of the clinical symptoms of PD.

In the present study, an MPTP-induced PD model was first established. The recognized neurobehavioral 

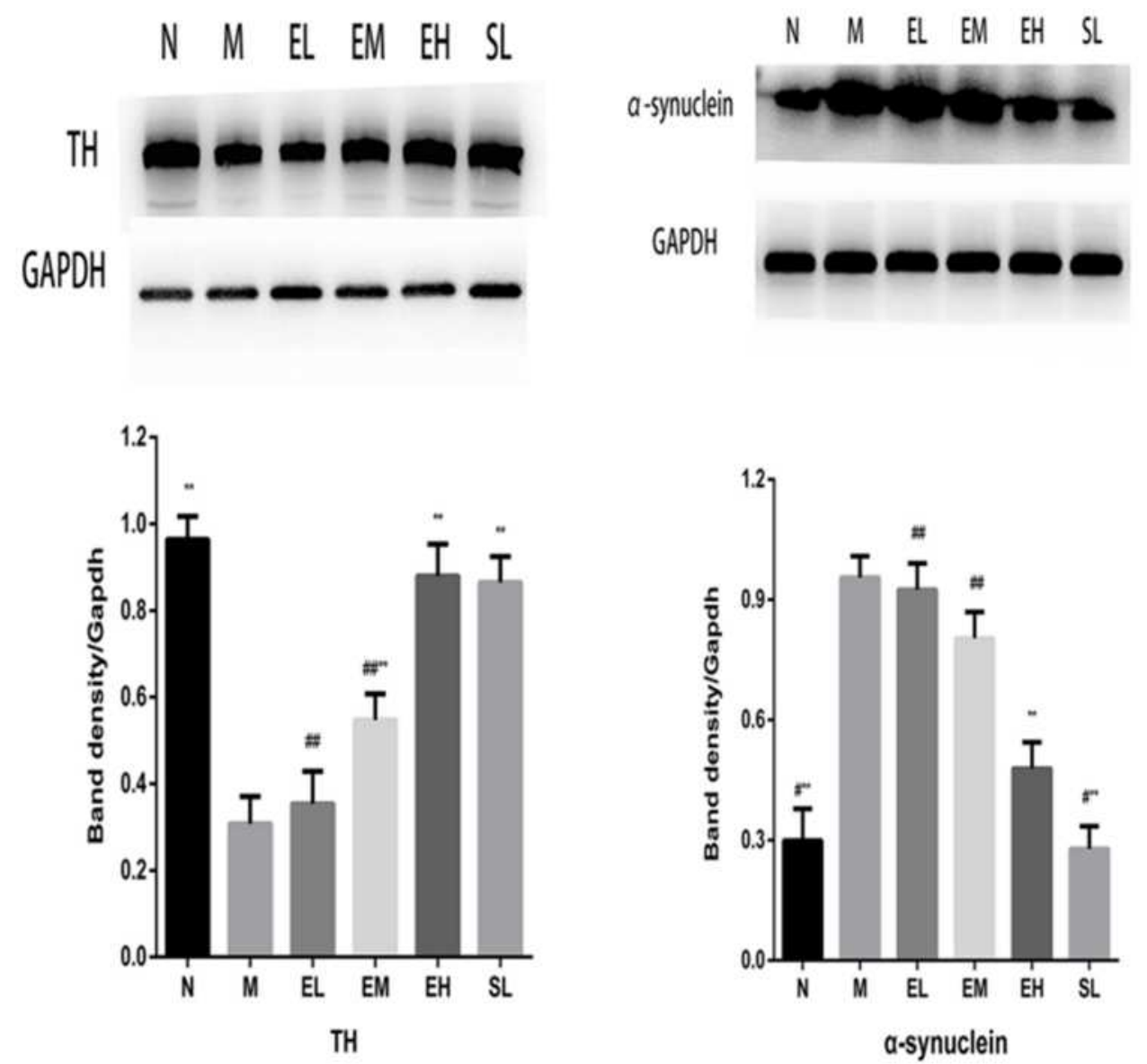

Figure 4 The increased protein expression of TH in the substantia nigra and decreased protein expression of $\alpha$-synuclein in the substantia nigra in MPTP-induced PD mice by echinacoside. ${ }^{* * \mathrm{P}}<0.0 \mathrm{I}$, compared with the model group; ${ }^{\#} \mathrm{P}<0.05,{ }^{\# \#} \mathrm{P}<0.0 \mathrm{I}$, compared with the high-dose echinacoside group.

evaluation methods, such as the pole test, the ability to engage in spontaneous activity, gait analysis, rotarod test, and other methods were adopted to conduct an in-depth evaluation of mice's neurobehavioral functioning. Compared with the MPTP group, mice in EM and EH groups exhibited significantly shorter pole-climbing time, significantly longer roller-residence time, and significantly longer fore and hind-limb strides. The spontaneous movement ability in mice also increased significantly. Accordingly, we believe that ECH can potentially significantly improve neurobehavioral functioning in PD mice.

Since $\mathrm{TH}$ is a unique enzyme of the dopaminergic neurons and the rate-limiting enzyme in DA synthesis, the expression of DA directly reflects the content of DA in the brain. ${ }^{15}$ The results of the present study confirmed that the expression of $\mathrm{TH}$ in $\mathrm{SN}$ in both $\mathrm{EM}$ and $\mathrm{EH}$ groups increased compared with the MPTP group; furthermore, a significant increase in the content of DA and DOPAC was observed in these two groups. Considering that the abnormal aggregation of $\alpha$-synuclein is the core pathological mechanism in $\mathrm{PD}$, immunohistochemistry and Western blot analysis were adopted to analyze the expression of $\alpha$-synuclein, which was reduced in the EH group.

In neurodegenerative diseases, dopaminergic neurons are more susceptible to injury due to oxidative stress, ${ }^{16}$ and oxidative stress can also lead to mitochondrial dysfunction and the accumulation of abnormal proteins. The latter two mechanisms are common causes of PD. ${ }^{17}$ Exposure to MPTP can cause a decline in the functioning of the endogenous antioxidant system, thereby increasing the sensitivity of the dopaminergic neurons to oxidative stress. The detection of MPTP-induced oxidative stress can be achieved by measuring SOD, CAT, GSH-Px, MDA, and other indicators. In a cell, when the superoxide is significantly increased, SOD will increase. ${ }^{18}$ Catalase is a specific enzyme that can detoxify reactive oxygen species (ROS) and remove $\mathrm{H}_{2} \mathrm{O}_{2}{ }^{19}$ When both SOD and CAT increase, the body will have a degree of defense against the release of free radicals 
A

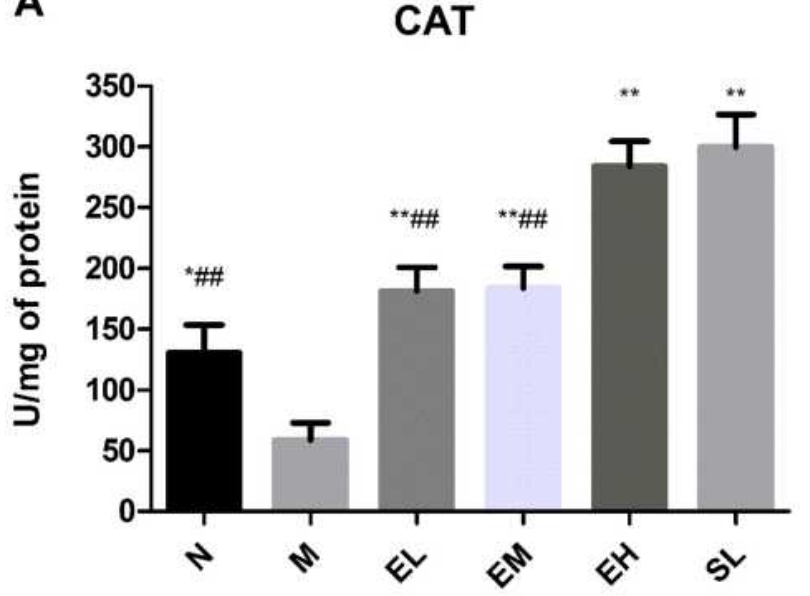

C

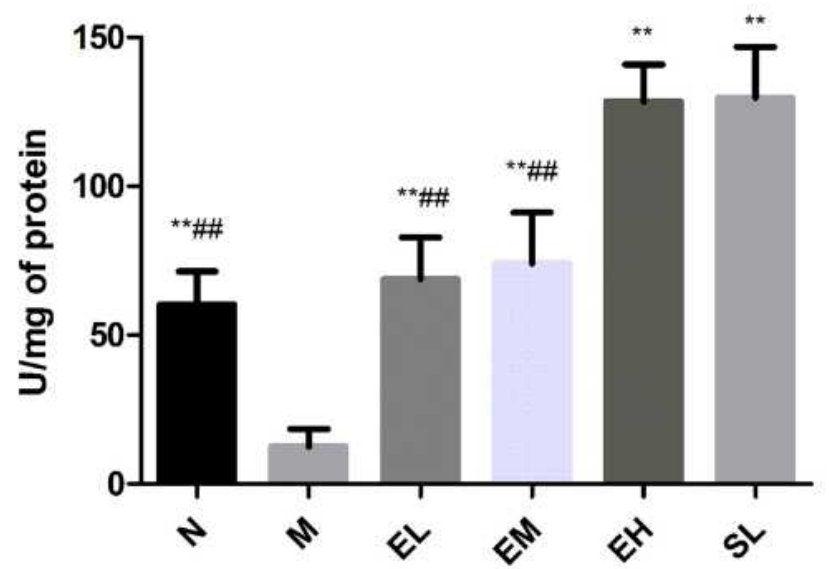

B

GSH-PX

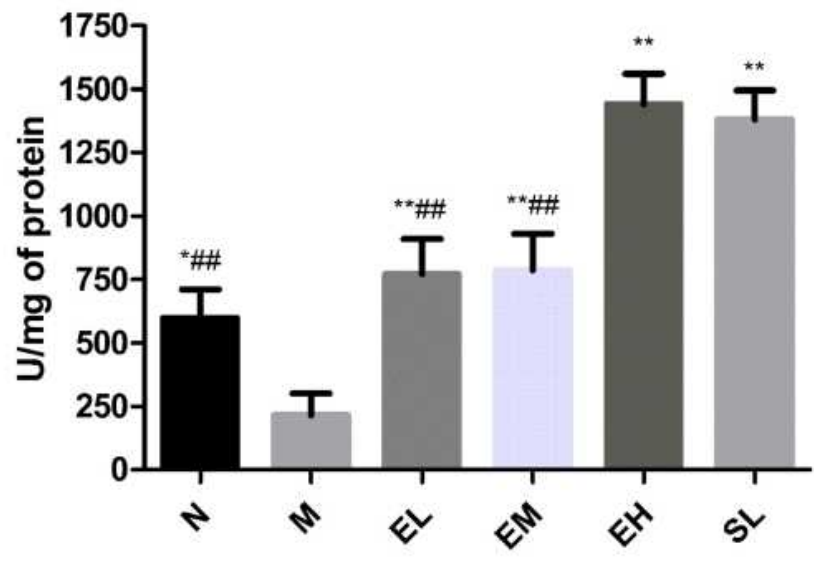

D

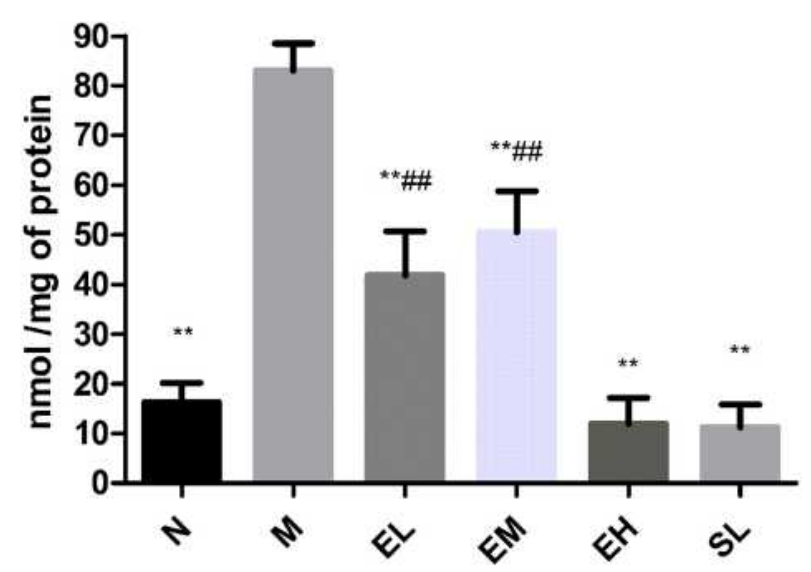

Figure 5 The effect of echinacoside on oxidative stress in the midbrain substantia nigra in MPTP-induced PD mice. (A) CAT; (B) GSH-PX; (C) SOD; (D) MDA. *P < 0.05 , $* * \mathrm{P}<0.0$ I, compared with the model group; ${ }^{\# \#} \mathrm{P}<0.0$ l, compared with the high-dose echinacoside group.

following an injury to the mitochondrial respiratory chain. Glutathione peroxidase is the main free radical scavenger in the human body. The increased expression of GSH-Px in the PD model has a significant protective effect on injured dopaminergic neurons. Maintaining GSH-Px viability under oxidative stress is crucial for cell survival. ${ }^{20}$ As a metabolite of lipid peroxidation reaction between oxygen free radicals and unsaturated fatty acids in biomembranes, changes in MDA content can reflect the degree of lipid peroxidation in tissue, as well as changes in the content of oxygen free radicals. ${ }^{21}$ Malondialdehyde is widely used in cell and animal experiments to evaluate lipid peroxidation. ${ }^{22}$ Studies have confirmed that $\mathrm{ECH}$ has significant free radical scavenging activity and anti-lipid peroxidation effects.
It was found that several phenolic hydroxyl groups in the molecule positively correlated with the antioxidant capacity. $^{23,24}$ According to recent research reports, ECH significantly reduces the generation of ROS induced by $\mathrm{MPP}^{+}$, thereby increasing cell survival. ${ }^{9}$ The present study found that the contents of SOD, CAT, and GSHPx in the MPTP group were significantly lower than those in the normal saline group; however, the MDA content was significantly higher than that in the normal saline group. The content of antioxidants SOD, CAT, and GSH-Px significantly increased in EL, EM, and $\mathrm{EH}$ groups, while the MDA content was significantly reduced. This was consistent with results reported in the 


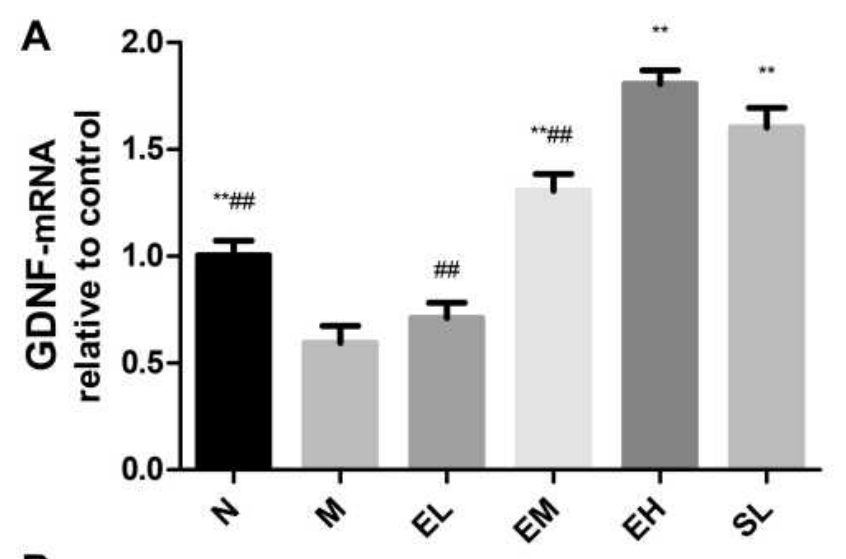

B

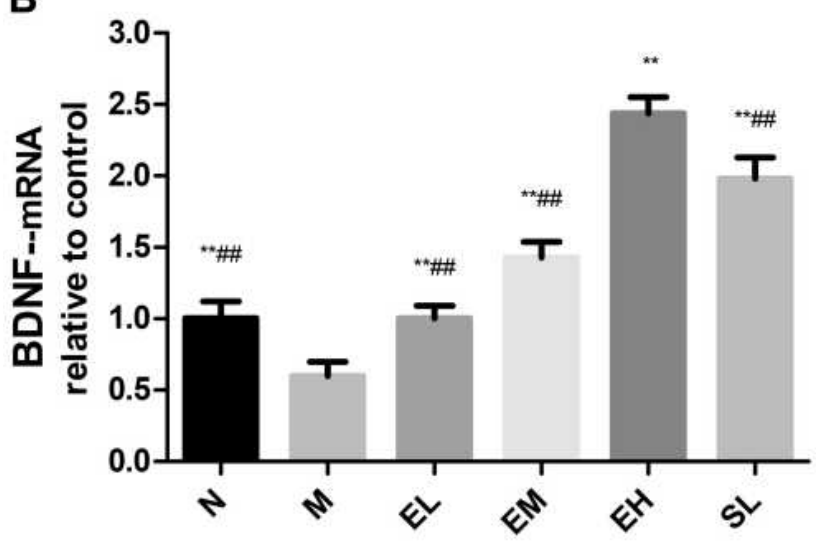

Figure 6 The increased mRNA expression of GDNF and BDNF in the substantia nigra in MPTP-induced PD mice by echinacoside. (A) GDNF; (B) BDNF. **P $<0.0$, compared with the model group; ${ }^{\#} \mathrm{P}<0.01$, compared with the high-dose echinacoside group.

above literature, confirming that ECH may have a significant effect on anti-oxidative stress in MPTPinduced PD mice.

It is known that GDNF and BDNF are the two most important neurotrophic factors for treating degenerative diseases of the nervous system, particularly PD. ${ }^{25}$ Although the effectiveness of neurotrophic factors in the treatment of PD has been confirmed in cell and animal models, it is difficult for the neurotrophic factor to pass through the blood-brain barrier because it is a macromolecular peptide. Moreover, because of several issues, such as infection, immune response, and unstable efficacy, in the administration modes of these drugs, their widespread application cannot be extended to clinical practice. ${ }^{26}$ Therefore, the development/search for a drug that can express or increase endogenous neurotrophic factors may have an encouraging effect on improving PD symptoms and even delay the progression of the disease. The present study found that the expression of GDNF and BDNF significantly increased in both EM and the EH groups. This was consistent with the results of the expression of $\mathrm{TH}$ in $\mathrm{SN}$, and of DA in the striatum among the two groups. It was also suggested that high expression of neurotrophic factors promoted the survival of dopaminergic neurons in SN and striatum. The experimental results of the present study were consistent with existing experimental results conducted by our research group, ${ }^{6,9,27,28}$ thereby further indicating that the neuroprotective effect of $\mathrm{ECH}$ may be closely correlated with the promotion of the high expression of neurotrophic factors.

In the present study, the common pro-inflammatory factors IL-6, TNF- $\alpha$, and anti-inflammatory factor IL-10 in $\mathrm{SN}$ in mice were measured by q-PCR in all groups. The results showed that the expression of IL-6 and TNF- $\alpha$ were significantly reduced in the EH group, together with an increase in the expression of IL-10, thus confirming that ECH may have an antiinflammatory effect. However, there were also limitations to the current study. We did not correlate the damage generally observed in the striatum with most of the proteins, and although we performed highperformance liquid chromatography for the striatum we did not adopt a similar approach for elements in the rest of the research.

\section{Conclusion}

In summary, the present study confirmed that oxidative stress, inflammation, and abnormal protein aggregation were involved in the pathogenesis of MPTP-induced PD models. Echinacoside may not only improve neurobehavioral symptoms in $\mathrm{PD}$ mice but also significantly increase the expression of $\mathrm{TH}$ in $\mathrm{SN}$ and $\mathrm{DA}$ in the striatum. The neuroprotective effect of $\mathrm{ECH}$ can potentially be correlated with anti-inflammation and antioxidation effects and promoting the expression of endogenous nerve growth factor. Additionally, the present study found that the accumulation of $\alpha$-synuclein (the core pathological product in PD) had been significantly reduced in the $\mathrm{EH}$ group. Because it is relatively economical, effective, and safe, ECH may provide a new therapeutic strategy for PD and even for other neurodegenerative illnesses including Alzheimer's disease. 
A

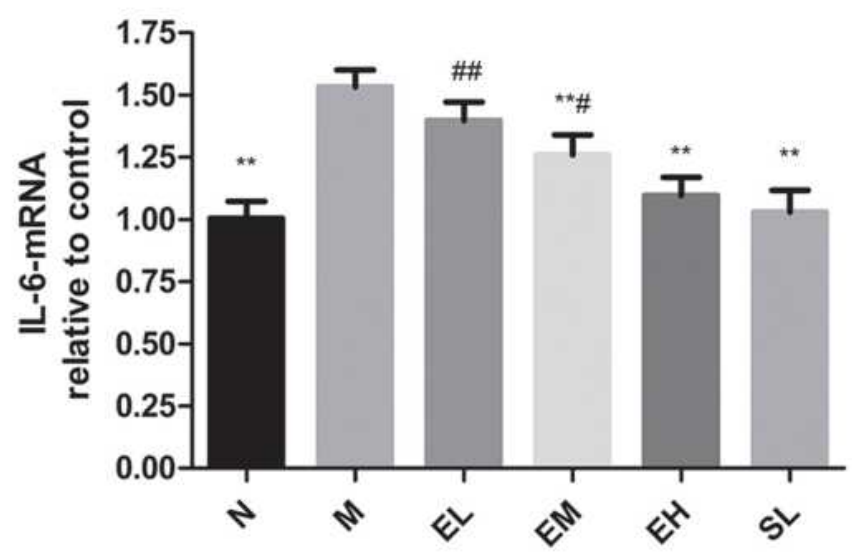

C

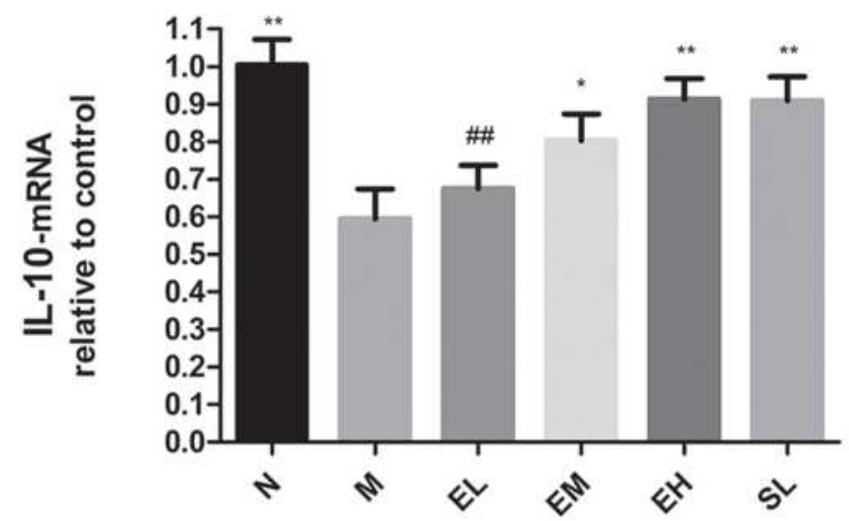

B

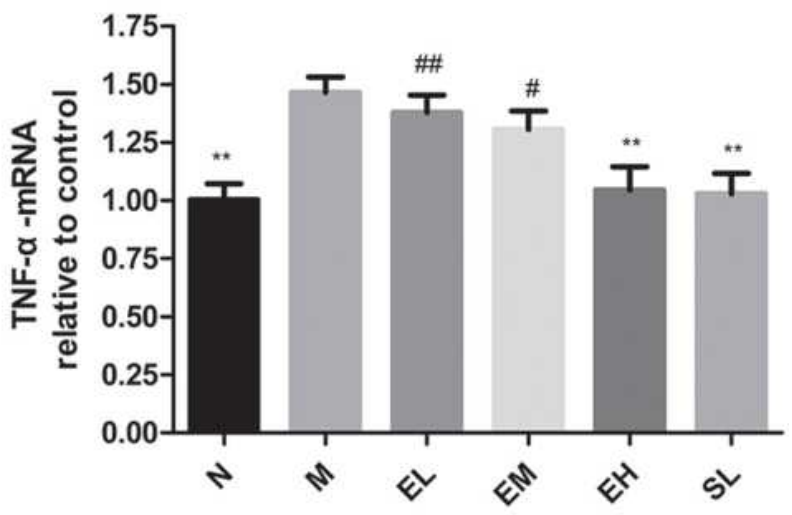

Figure 7 The reduced mRNA expression of inflammatory factors in the substantia nigra in MPTP-induced PD mice by echinacoside. (A) IL-6; (B) TNF- $\alpha$; (C) IL-I0. *P < 0.05 , ${ }^{* * P}<0.01$, compared with the model group; ${ }^{\#} \mathrm{P}<0.05,{ }^{\#} \mathrm{P}<0.01$, compared with the high-dose echinacoside group.

\section{Ethics Approval}

All experiments were evaluated and approved by the Eethics Committee of Nanjing Hospital of Chinese Medicine and complied with the National Institutes of Health Guide for the Care and Use of Laboratory Animals (KY2018027).

\section{Acknowledgments}

We are particularly grateful to all the people who have given us help with our article.

\section{Funding}

This study was funded by:Nanjing Health Science and Technology Development Special Fund Grant (YKK18134); Jiangsu Provincial Bureau of Traditional
Chinese Medicine "Chinese Medicine Brain Diseases" Key Discipline Cultivation Project (NBPY201704); Nanjing University of Chinese Medicine "Jiangsu University Nursing Advantageous Discipline Construction Project Funding Project" (2019YSHL098); Nanjing "Thirteenth Five-Year Plan" Famous Chinese Medicine Doctor (Kong-Jiang Liu) Studio Construction Project (LKJ-2017-NJ);Nanjing "Thirteenth Five-Year Plan" Famous Chinese Medicine Doctor (Jing-Qing Wang) Studio Construction Project (WJQ-2019-NJ).The funding body had no role in the design of the study and collection, analysis, and interpretation of data and in writing the manuscript.

\section{Disclosure}

The authors declare that they have no competing interests. 


\section{References}

1. Betarbet R, Sherer TB, MacKenzie G, Garcia-Osuna M, Panov AV, Greenamyre JT. Chronic systemic pesticide exposure reproduces features of Parkinson's disease. Nat Neurosci. 2000;3 (12):1301-1306. doi:10.1038/81834

2. Dunnett SB, Björklund A. Prospects for new restorative and neuroprotective treatments in Parkinson's disease. Nature. 1999;399(6738 Suppl):A32-A39. doi:10.1038/399a032

3. Parihar MS, Parihar A, Fujita M, Hashimoto M, Ghafourifar P. Mitochondrial association of alpha-synuclein causes oxidative stress. Cell Mol Life Sci. 2008;65(7-8):1272-1284. doi:10.1007/ s00018-008-7589-1

4. Liu M, He ZL. The evidence on dopamine receptor agonists in the treatment of Parkinson's disease. Chin J Evid Based Med. 2010;10 (03):232-234. Article in Chinese.

5. He WJ, Fang TH, Tu PF. Research progress on pharmacological activities of echinacoside. Zhongguo Zhong Yao Za Zhi. 2009;34 (04):476-479. Article in Chinese.

6. Zhao Q, Gao J, Li W, Cai D. Neurotrophic and neurorescue effects of echinacoside in the subacute MPTP mouse model of Parkinson's disease. Brain Res. 2010;1346:224-236. doi:10.1016/j. brainres.2010.05.018

7. Zhu M, Zhou M, Shi Y, Li WW. Effects of echinacoside on MPP $(+)$-induced mitochondrial fragmentation, mitophagy and cell apoptosis in SH-SY5Y cells. Zhong Xi Yi Jie He Xue Bao. 2012;10 (12):1427-1432. doi:10.3736/jcim20121215

8. Zhang J, Zhang Z, Xiang J, et al. Neuroprotective effects of echinacoside on regulating the stress-active p38MAPK and NF- $\kappa B$ p52 signals in the mice model of Parkinson's disease. Neurochem Res. 2017;42 (4):975-985. doi:10.1007/s11064-016-2130-7

9. Zhao Q, Yang X, Cai D, et al. Echinacoside protects against MPP (+)-induced neuronal apoptosis via ROS/ATF3/CHOP pathway regulation. Neurosci Bull. 2016;32(4):349-362. doi:10.1007/s12264016-0047-4

10. Chen C, Xia B, Tang L, et al. Echinacoside protects against MPTP/ MPP+-induced neurotoxicity via regulating autophagy pathway mediated by Sirt1. Metab Brain Dis. 2019;34(1):203-212. doi:10.1007/s11011-018-0330-3

11. Matsuura K, Kabuto H, Makino H, Ogawa N. Pole test is a useful method for evaluating the mouse movement disorder caused by striatal dopamine depletion. J Neurosci Methods. 1997;73(1):45-48. doi:10.1016/s0165-0270(96)02211-x

12. Sedelis M, Schwarting RK, Huston JP. Behavioral phenotyping of the MPTP mouse model of Parkinson's disease. Behav Brain Res. 2001;125(1-2):109-125. doi:10.1016/s0166-4328(01)00309-6

13. Byler SL, Boehm GW, Karp JD, et al. Systemic lipopolysaccharide plus MPTP as a model of dopamine loss and gait instability in C57B1/ 6J mice. Behav Brain Res. 2009;198(2):434-439. doi:10.1016/j. bbr.2008.11.027

14. Baquet ZC, Gorski JA, Jones KR. Early striatal dendrite deficits followed by neuron loss with advanced age in the absence of anterograde cortical brain-derived neurotrophic factor. $J$ Neurosci. 2004;24(17):4250-4258. doi:10.1523/JNEUROSCI.3920-03.2004
15. Yuan H, Sarre S, Ebinger G, Michotte Y. Histological, behavioural and neurochemical evaluation of medial forebrain bundle and striatal 6-OHDA lesions as rat models of Parkinson's disease. $J$ Neurosci Methods. 2005;144(1):35-45. doi:10.1016/j.jneumeth.2004.10.004

16. Cui H, Kong Y, Zhang H. Oxidative stress, mitochondrial dysfunction, and aging. J Signal Transduct. 2012;2012:646354. doi:10.1155/ 2012/646354

17. Perier C, Vila M. Mitochondrial biology and Parkinson's disease. Cold Spring Harb Perspect Med. 2012;2(2):a009332. doi:10.1101/ cshperspect.a009332

18. Kar S, Kavdia M. Endothelial $\mathrm{NO}$ and $\mathrm{O}_{2} \cdot{ }^{-}$production rates differentially regulate oxidative, nitroxidative, and nitrosative stress in the microcirculation. Free Radic Biol Med. 2013;63:161-174. doi:10.1016/j.freeradbiomed.2013.04.024

19. Ribeiro LC, Rodrigues L, Quincozes-Santos A, et al. Caloric restriction improves basal redox parameters in hippocampus and cerebral cortex of Wistar rats. Brain Res. 2012;1472:11-19. doi:10.1016/j. brainres.2012.07.021

20. Dokic I, Hartmann C, Herold-Mende C, Régnier-Vigouroux A. Glutathione peroxidase 1 activity dictates the sensitivity of glioblastoma cells to oxidative stress. Glia. 2012;60(11):1785-1800. doi:10.1002/glia.22397

21. Wang XF, Sun SG. Investigation of neuroprotective effect of topiramate on dopaminergic neuron. Stroke Nervous Dis. 2007;14(6):365. Article in Chinese.

22. Dalle-Donne I, Rossi R, Colombo R, Giustarini D, Milzani A. Biomarkers of oxidative damage in human disease. Clin Chem. 2006;52(4):601-623. doi:10.1373/clinchem.2005.061408

23. Xiong Q, Kadota S, Tani T, Namba T. Antioxidative effects of phenylethanoids from Cistanche deserticola. Biol Pharm Bull. 1996;19(12):1580-1585. doi:10.1248/bpb.19.1580

24. Mutrev G, Lei L, Tu PF, Guo DA, Lu JF. STUDY ON MOLECULAR MECHANISM OF ECHINACOSIDE FOR AGAINST AGING. Acta Biophys Sin. 2004;(03):183-187. Article in Chinese. https://kns.cnki.net $/ \mathrm{kcms} /$ detail $/$ detail.aspx? dbcode $=$ CJFD\&dbname $=$ CJFD2004\&filename $=$ SWWL200403003\&v $=$ P881ePJq0UDuGkffykLmx1V7vo4hyy3UmNhYPHGRYv4CBTctxL Lr9nHIjmodlTPe.

25. Wang J, Gallagher D, DeVito LM, et al. Metformin activates an atypical PKC-CBP pathway to promote neurogenesis and enhance spatial memory formation. Cell Stem Cell. 2012;11(1):23-35. doi:10.1016/j.stem.2012.03.016

26. Mochizuki H. Current status of gene therapy for Parkinson disease. Brain Nerve. 2009;61(4):485-493.

27. Zhao Q, Cai D, Bai Y. Selegiline rescues gait deficits and the loss of dopaminergic neurons in a subacute MPTP mouse model of Parkinson's disease. Int $J$ Mol Med. 2013;32(4):883-891. doi:10.3892/ijmm.2013.1450

28. Wang YH, Xuan ZH, Tian S, Du GH. Echinacoside Protects against 6-hydroxydopamine-induced mitochondrial dysfunction and inflammatory responses in $\mathrm{PC} 12$ cells via reducing ROS production. Evid Based Complement Alternat Med. 2015;2015:189239. doi:10.1155/ 2015/189239
Neuropsychiatric Disease and Treatment

\section{Publish your work in this journal}

Neuropsychiatric Disease and Treatment is an international, peerreviewed journal of clinical therapeutics and pharmacology focusing on concise rapid reporting of clinical or pre-clinical studies on a range of neuropsychiatric and neurological disorders. This journal is indexed on PubMed Central, the 'PsycINFO' database and CAS, and is the official journal of The International Neuropsychiatric Association (INA). The manuscript management system is completely online and includes a very quick and fair peer-review system, which is all easy to use. Visit http://www.dovepress.com/testimonials.php to read real quotes from published authors. 